\title{
Grain boundary energy anisotropy: a review
}

\author{
Gregory S. Rohrer
}

Received: 29 April 2011/Accepted: 30 May 2011/Published online: 10 June 2011

(C) Springer Science+Business Media, LLC 2011

\begin{abstract}
This paper reviews findings on the anisotropy of the grain boundary energies. After introducing the basic concepts, there is a discussion of fundamental models used to understand and predict grain boundary energy anisotropy. Experimental methods for measuring the grain boundary energy anisotropy, all of which involve application of the Herring equation, are then briefly described. The next section reviews and compares the results of measurements and model calculations with the goal of identifying generally applicable characteristics. This is followed by a brief discussion of the role of grain boundary energies in nucleating discontinuous transitions in grain boundary structure and chemistry, known as complexion transitions. The review ends with some questions to be addressed by future research and a summary of what is known about grain boundary energy anisotropy.
\end{abstract}

\section{Introduction}

The vast majority of the solid materials used in engineered systems are polycrystalline. In other words, they are comprised of many single crystals joined together by a three-dimensional (3D) network of internal interfaces called grain boundaries. Because the performance and integrity of a material are often determined by the structure of this network, grain boundaries have been of interest to materials scientists for many decades. There have been several recent review articles surveying grain boundary phenomena [1-5]. The current review is more narrowly

G. S. Rohrer ( $\square)$

Department of Materials Science and Engineering, Carnegie

Mellon University, Pittsburgh, PA 15213-3890, USA

e-mail: rohrer@cmu.edu focused on the topic of grain boundary energy anisotropy and provides an account of advancements since the publication of Sutton and Balluffi's [6] critical review of grain boundary energy data in 1987 . The paper contains a brief survey of historical concepts and grain boundary energy measurements. Next, findings from experiments and simulations are reviewed. This is followed by an introduction to recent findings about complexion transitions at grain boundaries. The review concludes with a prospectus for future studies of grain boundary energy anisotropy and a summary.

Grain boundaries are defects that have an excess free energy per unit area. This is evident by the fact that during most thermal and chemical etching processes, material near the grain boundary is preferentially removed. If a solid surface is polished and then etched, the preferential removal of material near the grain boundaries reveals the interfacial network, as illustrated in Fig. 1. To make a rough estimate for the average excess energy of a grain boundary, we can imagine building the interface by first creating two free surfaces and then joining them together to form the boundary. The energy to create the two surfaces will be twice the surface energy, $2 \gamma_{\mathrm{s}}$. However, the grain boundary energy will be less than this because of the binding energy $(B)$ gained when the two surfaces are brought together and new bonds are formed. The grain boundary energy is then:

$\gamma_{\mathrm{gb}}=2 \gamma_{\mathrm{s}}-B$

If we guess that the bonding at the interface restores one-half to three-quarters of the bonds to each side, then $\gamma_{\mathrm{s}} / 2 \leq B \leq 3 \gamma_{\mathrm{s}} / 4$ and $\gamma_{\mathrm{s}} / 2 \leq \gamma_{\mathrm{gb}}<\gamma_{\mathrm{s}}$. Using some simple approximations, Mullins [7] estimated $\gamma_{\mathrm{s}}$ as the elastic work done to create a free surface and found it to be $(E / 8) \times 10^{-10} \mathrm{~m}$, where $E$ is the elastic modulus. 


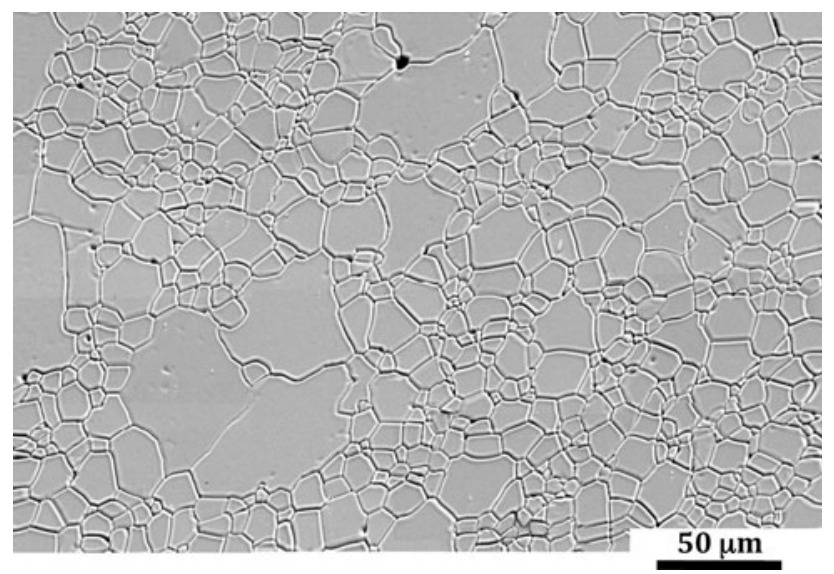

Fig. 1 A montage of secondary electron microscope images of spinel $\left(\mathrm{MgAl}_{2} \mathrm{O}_{4}\right)$ after thermal etching. During the anneal, material diffuses away from the boundary and the topography creates contrast in the image marking the locations where grain boundaries intersect the surface

Assuming an elastic modulus of $80 \mathrm{GPa}$, the resulting surface energy is approximately $1 \mathrm{~J} / \mathrm{m}^{2}$ and the average grain boundary energy $\left(\gamma_{\mathrm{gb}}\right)$ should then be in the range of $0.5-1.0 \mathrm{~J} / \mathrm{m}^{2}$. According to this estimate, the grain boundary energy should scale with the stiffness of the material.

The excess energy of the grain boundary provides the driving force for grain growth [8]. As grains shrink and disappear, the average grain size increases and the total grain boundary area per volume decreases. The capillary driving force, $2 \gamma_{\mathrm{gb}} /\langle r\rangle$, where $\langle r\rangle$ is a characteristic grain radius, decreases as $\langle r\rangle$ increases. Thus, as the average grain size increases, the driving force diminishes and it is more and more difficult to eliminate additional grain boundaries. This is why grain boundaries are nearly always found in solid materials, even though they are non-equilibrium defects.

The paragraphs above refer to an average grain boundary energy, but this review focuses on the anisotropy of the grain boundary energy. The anisotropic characteristics of the energy have been recognized since at least the time of Smith [9] and it has recently been shown that the probability that a grain boundary is annihilated during grain growth is related to its energy, and this leads to an anisotropic distribution of grain boundary types [10]. The energy anisotropy arises because different grain boundaries have different microscopic structures; following the line of reasoning that leads to Eq. 1, anisotropy in the grain boundary energy can arise from either $\gamma_{\mathrm{s}}$ or $B$. Macroscopically observable crystallographic parameters are used to classify boundaries with different microscopic structures. To classify the boundaries, five independent parameters must be specified. Three describe the misorientation of the crystal lattice and two describe the orientation of the grain boundary plane. The implication of having five independent parameters is that the number of different grain boundary types is large [4]. If the five dimensional domain of grain boundary types is discretized in $10^{\circ}$ intervals, then there are roughly $6 \times 10^{3}$ different grain boundaries for a material with cubic symmetry. The number of distinct boundaries increases rapidly for finer discretizations and for crystals with reduced symmetry.

Throughout this review, the so-called "axis-angle" description will be used to specify the three parameters of grain boundary lattice misorientation, $\Delta g$. In other words, a misorientation will be specified by a crystallographic axis common to both crystals, $[u v w]$, and a rotation about that axis, $\theta$. The grain boundary plane orientation is specified by the unit vector, $\mathbf{n}$. While $\mathbf{n}$ can assume any orientation within a hemisphere, certain special grain boundary plane orientations are sometimes referred to with the terms "tilt" and "twist". For any lattice misorientation, the twist boundary is the one for which $[u v w]$ and $\mathbf{n}$ are parallel. The tilt grain boundaries are those for which $\mathbf{n}$ is perpendicular to $[u v w]$. The term "symmetric" tilt means that the crystallographic planes bounding the grains on each side of the boundary are identical. All other tilts are asymmetric. The grain boundary character distribution (GBCD) is defined as the relative areas of grain boundaries as a function lattice misorientation and grain boundary plane orientation, $\lambda(\Delta g$, n). Analogously, the grain boundary energy distribution (GBED) is defined as the relative energies of grain boundaries as a function lattice misorientation and grain boundary plane orientation, $\gamma_{\mathrm{gb}}(\Delta g, \mathbf{n})$.

\section{Historical concepts for grain boundary structure and energy}

The earliest speculation on the microscopic structure of grain boundaries dates back the "amorphous cement" model put forth by Rosenhain and Ewen [11] in 1912. To fully appreciate this model, it is important to note that the year it was published was the same year that X-ray diffraction was invented. Therefore, when the model was conceived, the atomic structures of crystals had yet to be determined. It was already assumed that crystals were built from regular, periodically repeating "crystal units", like bricks, that were comprised of an as yet unknown group of atoms or molecular species. In the modern context, we can think of these building blocks as unit cells. Rosenhain and Ewen [11] imagined that when a boundary between two misoriented crystals was formed, the crystal units impinged at points and left interstitial space. These interstices were filled by "liquid molecules," which are the fundamental units (atoms or molecules) that make up the crystal unit. The rationale was that when the interstitial spaces were smaller than a crystal unit, the atoms or molecules would 
be unable to aggregate and, not crystallizing, would remain amorphous. The uncrystallized material was referred to as an "amorphous cement" that held the boundary together. This model is illustrated schematically in Fig. 2a.

Many years later, having knowledge of the atomic structure of crystals, Hargreaves and Hill [12] proposed that every atom in the boundary region could be associated with the crystal on one side or the other. Atoms in a transition zone (five to six plane on either side of the boundary, according to the illustration in their paper) near the grain boundary would be slightly displaced from their ideal positions (see Fig. 2c). More significantly, Hargreaves and Hill [12] also recognized the existence of coincidence boundaries, where some of the atomic sites in each crystal overlap within the boundary plane. They wrote an equation to find the rotational angles for different coincidence boundaries and illustrated a grain boundary where every fifth site was in coincidence (see Fig. 2b).

If we generalize these ideas using contemporary terminology, we can say that the Rosenhain and Ewen [11] model suggests that the atoms in the grain boundary region have a disordered structure that accommodates the orientation transition between the two crystals and the positions of these boundary atoms are not extensions of lattices of either of the adjoining crystals. The Hargreaves and Hill [12] model suggests more perfect order, with some relaxation in atomic positions, until an atomically abrupt transition. The two models could be characterized as a disordered boundary model and an ordered boundary model. In the past, ordered and disordered grain boundary models have been pitted against one another as if it must be one or the other. However, the current state of knowledge suggests that neither model is always a good description of a boundary. The increasingly powerful microscopic probes and computational models that have been applied to study grain boundaries have provided strong evidence for the existence of both ordered and disordered grain boundaries in polycrystals.

Read and Shockley [13] produced the first successful model to predict the energy anisotropy of grain boundaries. Their model, which is limited to grain boundaries with a relatively small misorientation angle, recognizes that lattice misorientations can be accommodated by dislocations. The basic idea is schematically illustrated for a tilt grain boundary in Fig. 3. As the lattice misorientation increases from zero, it is approximately proportional to the number of dislocations. As each dislocation carries a discrete amount of excess energy, the grain boundary energy is simply the sum of those dislocation energies plus the energy of interaction that arises as they are brought together to form the boundary. Therefore, as long as the dislocation spacing is large and they do not interact, the energy and misorientation are linearly proportional. When they are closer together, interactions among the dislocations will make the dependence of misorientation on energy more complex. The Read-Shockley [13] formula can be written in the following way:

$\gamma_{\mathrm{gb}}=E_{0} \theta[A-\ln \theta]$

where $E_{0}$ and $A$ have a dependence on the boundary plane orientation, but the principal variable is the misorientation angle, $\theta$ [13]. Gjostein and Rhines [14] measured the energies of tilt grain boundaries in $\mathrm{Cu}$ and found that for boundaries with $\theta<6^{\circ}$, agreement with Eq. 2 was reasonable, but at higher misorientation angles the theory underestimated the actual energy. At misorientations greater than $6^{\circ}$, the dislocation cores are separated by less than 10 atomic planes and the elastic theory applied to derive Eq. 2 is not expected to accurately depict the energy.

The Read-Shockley [13] model is widely accepted as providing a good explanation for the energies of low misorientation angle grain boundaries. Beyond this, the theory (a)

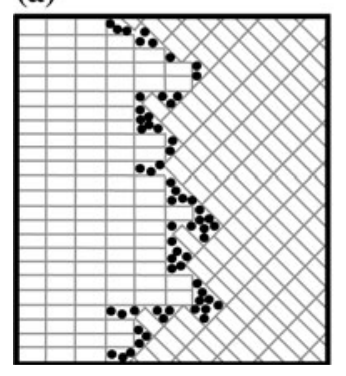

(b)

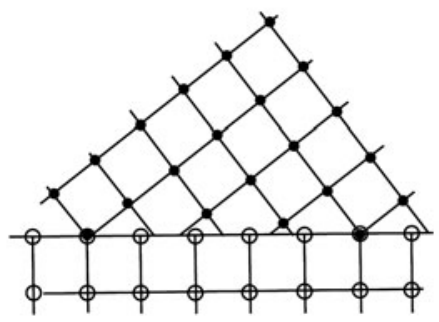

(c)

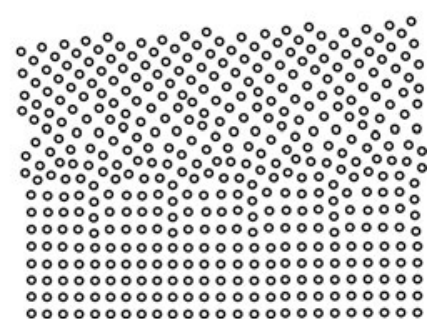

Fig. 2 Historical models for grain boundary structure. a The amorphous cement model where uncrystallized atoms (black dots) fill the interstices between misoriented grains formed by rigid crystal units (rectangles). Drawn after Figure 1 of Ref. [11]. b Ordered boundary model of Hargreaves and Hill [12], who pointed out that every fifth interface atom was in coincidence for a $36^{\circ}$ rotation about [100]. c Boundary transition region proposed by Hargreaves and Hill [12] who assumed that atoms in the vicinity of the boundary would adopt relaxed positions. b, c drawn after Figures 11 and 12 of Ref. [12] 


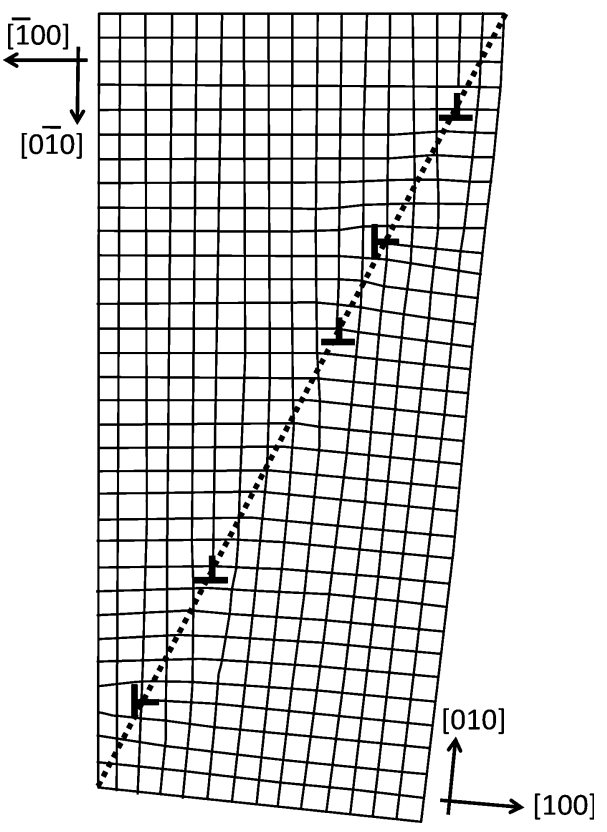

Fig. 3 Schematic of a low angle grain boundary comprised to two types of edge dislocations. The lattice for the crystal to the right of the dashed line (the grain boundary) is rotated with respect to the crystal on the left. Drawn after figure 12.1 in Ref. [16]

actually makes it possible to represent any possible grain boundary as a collection of dislocations. Given the lattice misorientation $(\Delta g)$, grain boundary plane orientation $(\mathbf{n})$, and three non-coplanar Burgers vectors, Frank's formula $[15,16]$ can be used to determine the density of these dislocations needed to create the boundary. In the simplest approximation, the boundary energy can be assumed to be proportional to the minimum geometrically necessary dislocation density.

Coincident site lattices (CSL) are one of the most influential concepts in the study of grain boundaries during the past 60 years. Kronberg and Wilson [17] noticed that certain grain boundary misorientations commonly found in copper after secondary recrystallization corresponded to lattice rotations that placed a fraction of the atomic sites in coincidence. Note that while the CSL concept is nearly always attributed to Kronberg and Wilson [17] in the materials science literature, the key concepts were first described by Friedel in 1920 [18, 19], and then independently by Hargreaves and Hill [12] in 1929. The potential importance of the boundaries was noted by Aust and Rutter [20], who reported that some high coincidence grain boundaries in $\mathrm{Pb}-\mathrm{Sn}$ alloys migrated at much faster rates than others. CSL misorientations are named by the inverse of the number of coincident sites. For example, for a twin in an fcc materials, one-third of the sites are in coincidence so this it known as a $\Sigma 3$ boundary. Because of this relationship, a smaller $\Sigma$ number is typically associated with special characteristics. As examples, four coincident site lattices obtained by rotations about the [100] axis in a cubic material are illustrated in Fig. 4.

The CSL concept does not explicitly address grain boundary energy and the authors of the earliest papers do not suggest that the special boundaries have low energy. However, a reduced grain boundary energy was suggested by Brandon [21, 22], who extended to high coincidence boundaries Read and Shockley's [13] concept of using dislocations to compensate small orientation differences. Using this concept, Brandon [21] was able to define an angular width for the assumed region of reduced grain boundary energy. While some coincident site boundaries clearly have lower energies than general boundaries (the $\Sigma 3$ twin is an example), the phenomenon is not general. For example, Goodhew et al. [23] found that the CSL concept could not explain the configuration of tilt grain boundaries in a gold foil and Chaudhari and Matthews [24] concluded that coincidence site density is not a good guide to the energies of coincidence boundaries in $\mathrm{MgO}$. In Sutton and Bulluffi's [6] seminal review of models for interfacial boundary energy, they concluded that there was "no support for the general usefulness of criteria" based on coincident site density.

Another relevant model for grain boundaries was developed based on the idea that grain boundary structures in simple metals could be represented by selected groups of eight fundamental polyhedra [25-27]. A two-dimensional example is shown in Fig. 5. The authors used hard sphere models to show that symmetric and asymmetric tilt and twist type grain boundaries can all be represented in this way. One of the advantages of this model is that it provides a simple description of the local atomic packing in the boundary, based on a small number of basic building blocks. Assuming that an energy can be assigned to each of the polyhedral building blocks, then the energy of any boundary can be approximated simply by summing the energies of the components. This has proven to be relevant in the interpretation of the energies of tilt grain boundaries in fcc metals [26].

Most calculations of grain boundary energies have been carried out over a relatively limited crystallographic domain. However, Wolf [28-31] was among the first to compute grain boundary energies using consistent methods over a broad crystallographic domain. The results indicated that the grain boundary energy depends strongly on the grain boundary plane; the energy as a function of misorientation angle for constant grain boundary planes is relatively smooth, as illustrated in Fig. 6, compared to differences between boundaries with different planes [31]. It was suggested that structural disorder in the interface enhances the anisotropies present in the free surface. 
Fig. 4 Coincident site lattice configurations $(\Sigma \leq 25)$ obtained by rotations of the blue cubic lattice about a common [001] axis normal to the plane of the paper. a $\Sigma 25$ at $16^{\circ}, \mathbf{b} \Sigma 13$ at $22^{\circ}$, c $\Sigma 17$ at $28^{\circ}$, and $\mathbf{d} \Sigma 5$ at $36^{\circ}$. The black lines are drawn to indicate the CSL repeat units
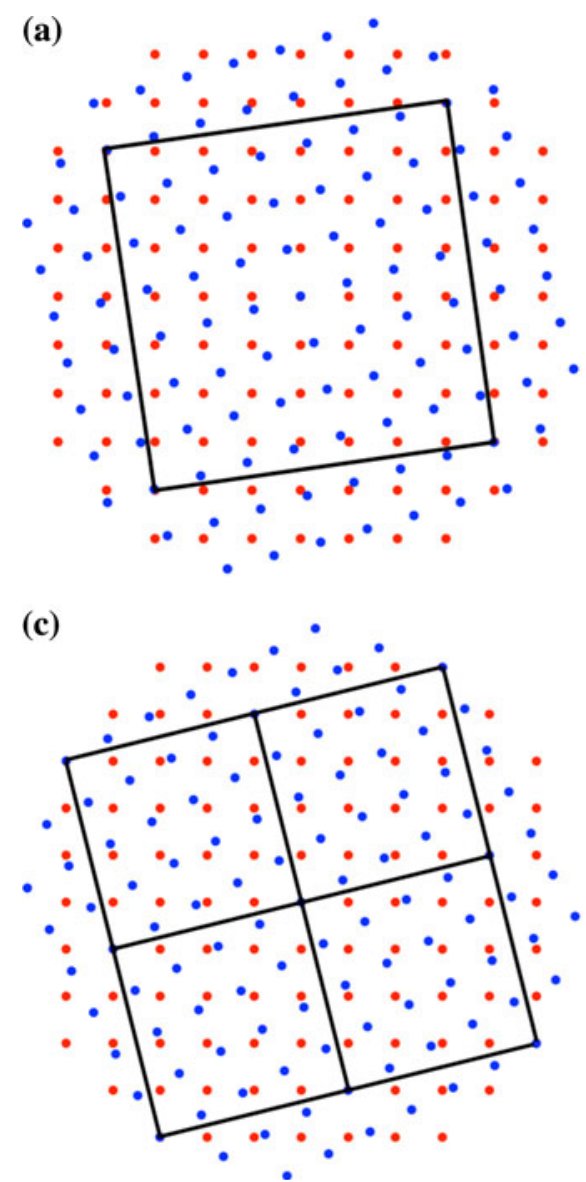

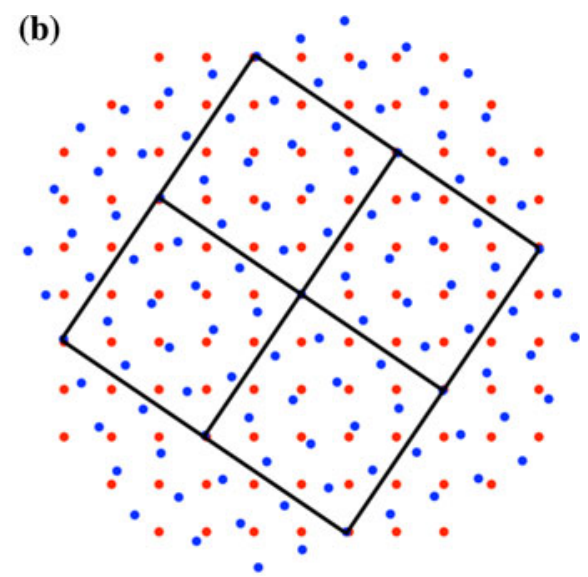

(d)

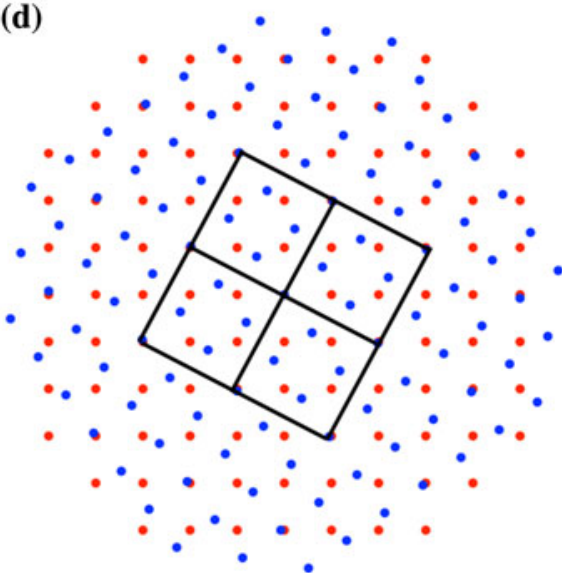

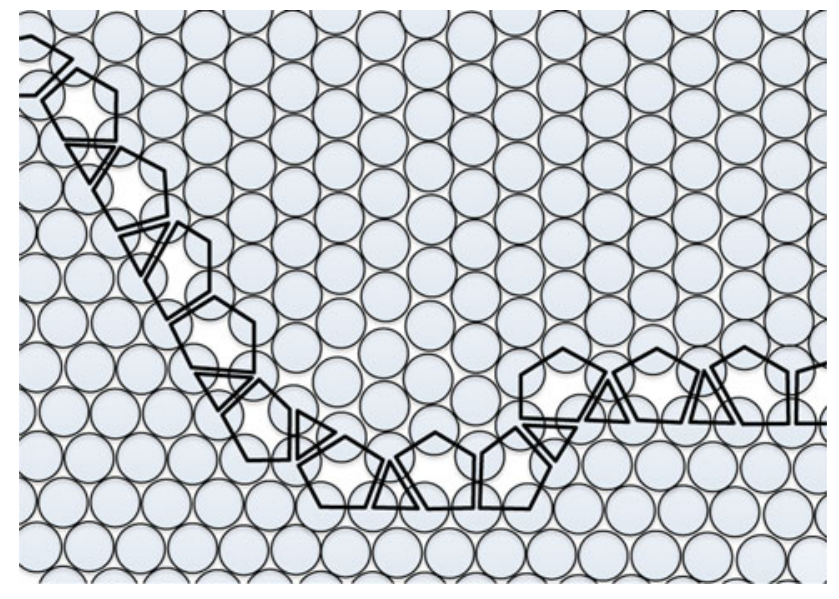

Fig. 5 Illustration of the polyhedral repeat unit model for a grain boundary between two closest packed hard sphere crystals. The boundary is comprised of pentagons and triangles. Drawn after Figure 6c of Ref. [25]

\section{Measuring grain boundary energies}

Grain boundary energy measurements are all carried out by observing the geometry of interface junctions assumed to be in thermodynamic equilibrium. Herring [32] described

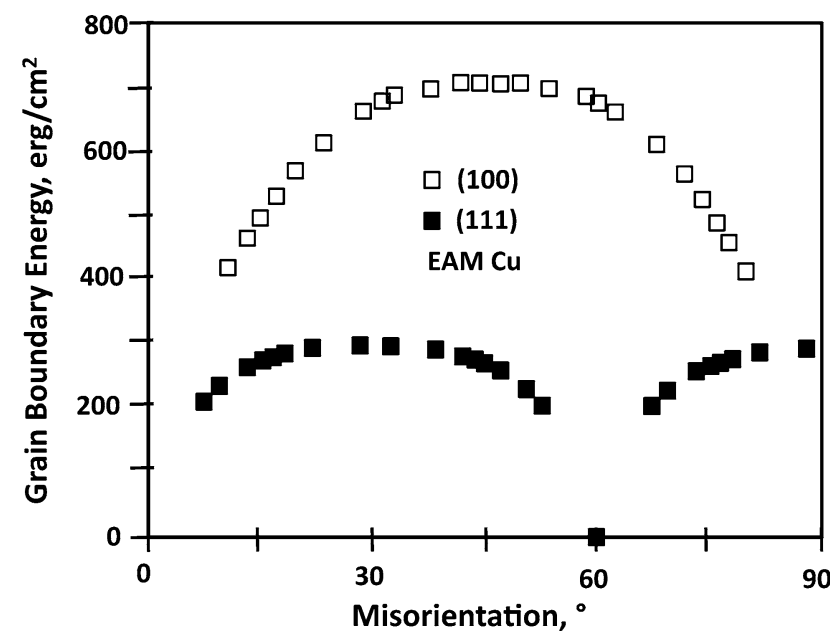

Fig. 6 The grain boundary energies versus twist angle for (100) and (111) twist boundaries, calculated for $\mathrm{Cu}$ using an embedded atom model potential. The very low energy for the $60^{\circ}(111)$ twist boundary corresponds to the coherent twin. Drawn after Figure 3a of Ref. [31]

the equilibrium between interfacial forces at a triple line in the following way:

$\gamma_{i} \vec{t}_{i}+\frac{\partial \gamma_{i}}{\partial \beta} \vec{n}_{i}=0$ 
Referring to Fig. 7a, Eq. 3 is a summation over the three interfaces where $\gamma_{i}$ is the energy of the $i$ th interface. This is a vector balance of forces tangential $\left(\vec{t}_{i}\right)$ and normal $\left(\vec{n}_{i}\right)$ to the interfaces, as labeled in Fig. 7a. The tangential forces, shown by solid lines, amount to a three-way tug-of-war at the triple junction. If one interface has a higher energy, it will pull the triple line along its tangent, annihilating the relatively higher energy interface and replacing it by the lower energy interfaces. The normal forces, shown by dashed lines and often referred to as torques, result from anisotropy. If the differential of the energy with respect to rotation angle $\beta$ is large, there is a normal force to rotate the boundary in the direction that lowers the energy. For the junction to be in equilibrium, the six forces must balance, and this is reflecting in Eq. 3.

The Herring equation can be simplified in several ways and most grain boundary energy measurements have been made using one of these simplifications. For example, if it is assumed that the differential terms are small enough to be ignored, then only the tangential forces need to be balanced. In this case, two equations can be written for the force balance in the perpendicular $x$ and $y$ directions. Solving the equations yields a simplified form of Eq. 3 usually referred to as Young's equation:

$\frac{\gamma_{1}}{\sin \theta_{2,3}}=\frac{\gamma_{2}}{\sin \theta_{1,3}}=\frac{\gamma_{3}}{\sin \theta_{1,2}}$

The terms in this equation are defined in Fig. 7b. This makes it possible to determine the relative grain boundary energy by measuring only the dihedral angles between crystals of known orientation. This equation has been used to determine the grain boundary energy anisotropy in an Fe-Si [33] alloy, $\mathrm{Al}[34,35]$, and $\mathrm{MgO}$ [36].

For the case where a grain boundary meets a free surface, the equation can be simplified further if it is assumed that the surfaces have the same energy. Using the geometry in Fig. 7c, the balance of vertical forces yield Mullins' equation [37] for the scale invariant geometry of a grain boundary thermal groove formed by surface diffusion:

$\frac{\gamma_{\mathrm{gb}}}{\gamma_{\mathrm{s}}}=2 \cos \left(\frac{\Psi}{2}\right)$

In this case, measurement of the grain boundary dihedral angle $(\Psi)$ permits an estimate of the grain boundary to surface energy ratio. In the earliest measurements, interference microscopy was used to measure $\Psi$. However, the more recent development of the atomic force microscope has made the measurement of $\Psi$ relatively easy [38].

While the simplified approaches have facilitated numerous measurements, it should be emphasized that it is not possible to evaluate the full anisotropy of the grain boundary energy without using the complete form of the Herring [32] equation. To do this, one needs to examine triple junctions involving all different types of grain boundaries. Because the necessary number of junctions is in the range of $10^{3}-10^{4}$, it was not feasible to conduct such measurements using manual techniques. However, the development of automated electron backscatter diffraction orientation mapping in the scanning electron microscope has made it possible to characterize the crystallography of $10^{4}-10^{5}$ triple junctions in a reasonable amount of time [39-41]. When coupled with serial sectioning, it is possible to determine the complete geometry for triple lines involving all boundary types and apply the Herring equation [42-44].

A method for doing this was developed by Moraweic [45]. The domain of grain boundary types is discretized so that there is a finite number of unknown boundary energies. The equilibrium condition in Eq. 3 can be written for each observed triple junction. As long as the number of (a)

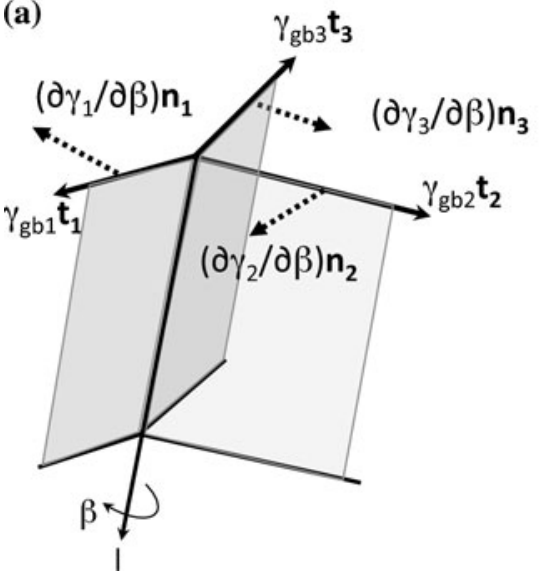

(b)

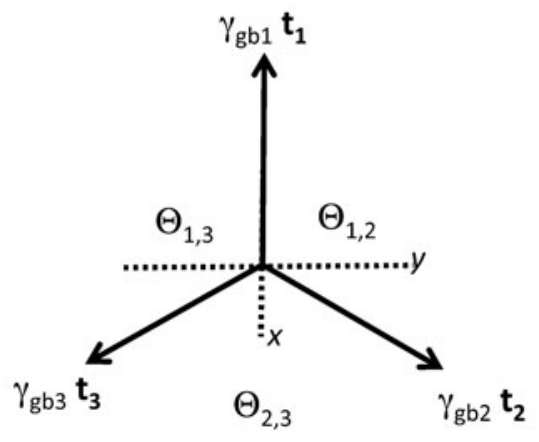

(c)

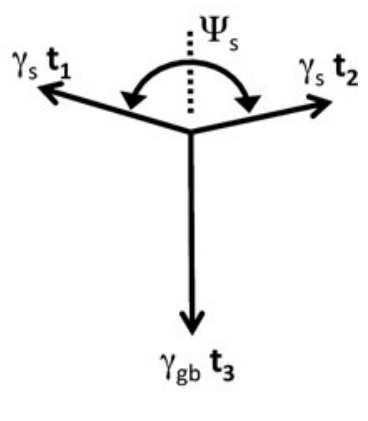

Fig. 7 Illustrations of the balance of interfacial energies at triple junctions, defining the quantities in Eqs. 3, 4, and 5. a Tangential and normal vectors in the Herring relation. $\mathbf{b}$ If the torque terms are ignored, only the tensions must balance. c The special case of the grain boundary thermal groove, where the surfaces are assumed to have the same energy 
equilibrium equations (observed triple junctions) exceeds the number of unknown grain boundary energies, it is possible to determine a set of energies that best satisfy the equations. The implementation of the method has been described in detail in Ref. [45] and has resulted in the determination of several complete grain boundary energy distributions from metals and ceramics [44, 46-48]. The method has also been applied to determine surface energy anisotropy $[49,50]$. The method is sensitive to the amount of data available, so the energies of the most rarely observed boundaries are the most uncertain. Furthermore, in places where the energy varies rapidly with angle, the depth of the minimum or height of the maximum will be underestimated.

\section{Measurements of grain boundary energy anisotropy}

We begin by reviewing the grain boundary energy anisotropies that have been measured using the tricrystal and thermal groove methods; in these cases, the differential terms in the Herring equation are neglected. We will concentrate on measurements where the grain boundary plane is controlled and known. Results for $\mathrm{Cu}$ and $\mathrm{Al}$ are compared in Fig. 8 because they both have the fcc structure $[14,34,35,51]$. In both cases, the metals were annealed very near the melting points, so the energy anisotropy reflects high temperature behavior. Figure 8a shows that in both $\mathrm{Al}$ and $\mathrm{Cu}$, the grain boundary energy varies smoothly with the misorientation angle for [100] symmetric tilt boundaries. Although there are boundaries of high coincidence in this series, their energies do not differ significantly from the boundaries without high coincidence. This result is consistent with measurements of the energies of [100] misorientation grain boundaries (of undetermined grain boundary plane orientation) in Inconel reported by Skidmore et al. [52].

Compared to the [100] tilt series, there is more variation in the energies for the [110] symmetric tilt grain boundaries. In Fig. 8b, data from Al [34], again very near the melting point, is compared to data for $\mathrm{Cu}$ at a range of temperatures [51]. All of the data agrees that for a $70^{\circ}$ rotation about [110] there is a deep minimum in the energy; this is the coherent twin. For $\mathrm{Al}$ and the $\mathrm{Cu}$ at the highest temperature, there is also agreement that there is a minimum at $130^{\circ}$ that corresponds to the $\Sigma 11$ (113) boundary. The low energy of this boundary also agrees with observations reported by McLean [53]. However, for $\mathrm{Cu}$ at lower temperatures, this minimum moves closer to the $\Sigma 9$ boundary at $140^{\circ}$ [51]. One significant difference between $\mathrm{Al}$ and $\mathrm{Cu}$ is that the ratio of the energies of the symmetric $\Sigma 9\left(\right.$ at $\left.40^{\circ}\right)$ and the coherent twin (at $70^{\circ}$ ) is much larger in $\mathrm{Cu}$ than $\mathrm{Al}$. Despite the differences, the similar overall
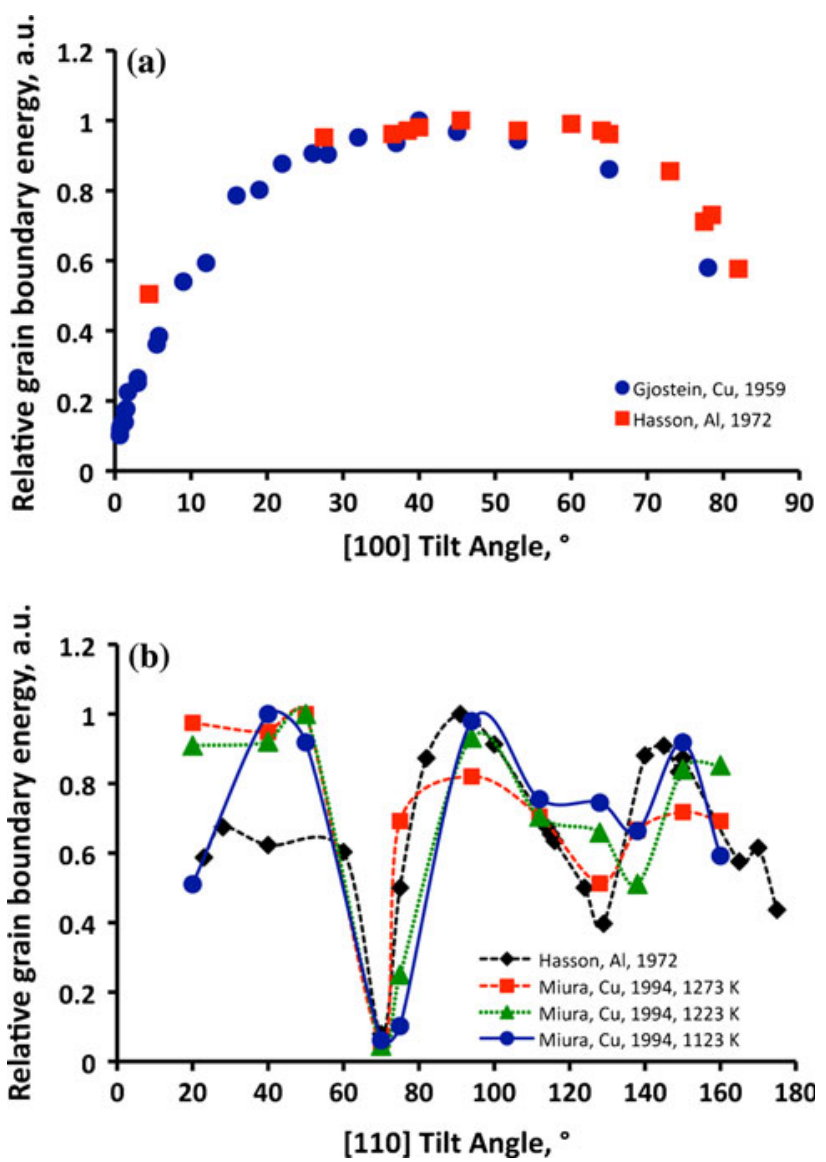

Fig. 8 Grain boundary energy data for fcc metals. For comparison, the maximum value in each data set was set to 1 . a Symmetric [100] tilt grain boundaries in $\mathrm{Al}[34,35]$ and $\mathrm{Cu}$ [14]. b Symmetric [110] tilt grain boundaries in $\mathrm{Al}[34,35]$ and $\mathrm{Cu}[51]$

appearance of the data indicates that the energy anisotropy might have a strong link to the ideal crystal structure. Finally, it should be noted that recent calculations of the energies of the symmetric tilt boundaries in $\mathrm{Cu}$ and $\mathrm{Al}$ agree well with the high temperature data [54].

The same techniques have been used to examine ceramic materials with similar results. For example, two sources reporting the energies of [100] symmetric tilt grain boundaries in $\mathrm{NiO}$ both show that it varies smoothly, as reported for $\mathrm{Cu}$ and $\mathrm{Al}$ (see Fig. 8a) [55, 56]. However, the relative energies for symmetric [110] tilt grain boundaries show more variation [57]. In Fig. 9a, measurements for $\mathrm{NiO}$ [57] and $\mathrm{MgO}$ [58], both of which have the rock salt structure, are compared. Both show a minimum at the position of the coherent $\Sigma 3$ twin. There are also weak minima at $\Sigma 9\left(40^{\circ}\right)$ and $\Sigma 11\left(130^{\circ}\right)$ in both data sets. In general, one might say the data sets are comparable, except for the fact that the lowest misorientation angle grain boundaries in $\mathrm{MgO}$ do not show the expected reduced energy. The [100] twist grain boundaries in $\mathrm{MgO}$ (see Fig. 9b) also show a strong variation in energy with 
misorientation angle, with minima at $\Sigma 17\left(28^{\circ}\right), \Sigma 13\left(22^{\circ}\right)$, and $\Sigma 5\left(36^{\circ}\right)$ [58].

Until recently, studies of grain boundary energy have been generally limited a small number of grain boundaries, as in the studies described above. Automated methods have made it possible to determine the energies of all possible grain boundaries, and this has led to a number of new insights [42-44, 47, 48, 59]. The first is that grain boundary energies are strongly dependent on the grain boundary plane orientation. In other words, when the misorientation of the grain boundary is fixed, some grain boundary plane orientations have significantly lower energies than others. This is illustrated in Fig. 10, which compares the grain boundary energy distributions in $\mathrm{MgO}$ with the grain boundary character distributions [43, 44]. The stereograms in Fig. 10a-c show the energy as a function of grain boundary plane orientation at three fixed misorientations and there are distinct minima at (100) orientations. The stereograms in Fig. 10d-f show the relative areas of grain boundaries as a function of grain boundary plane
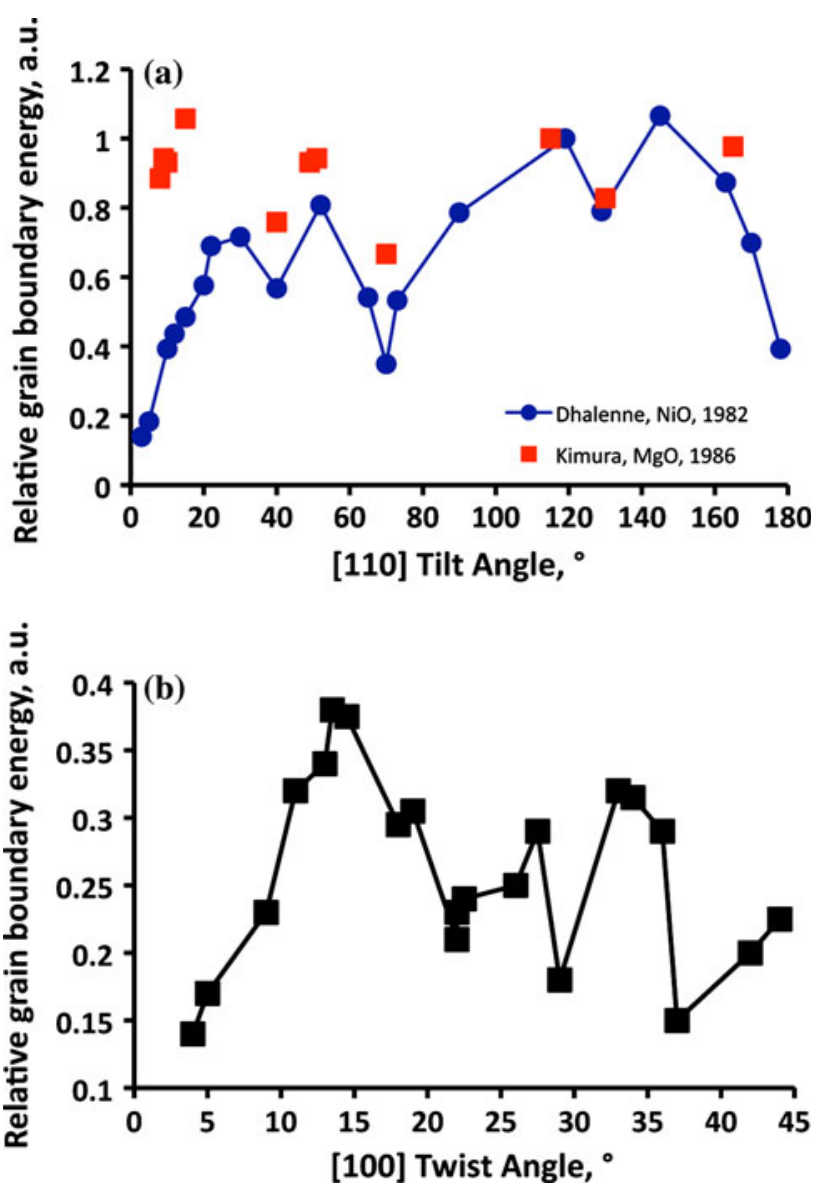

Fig. 9 Grain boundary energy data for oxides with the rock salt structure. a The relative energies of symmetric [110] tilt grain boundaries in $\mathrm{MgO}$ [58] and $\mathrm{NiO}$ [57]. For comparison, the maximum value in each data set was set to 1 . b The relative energies of [100] twist grain boundaries in $\mathrm{MgO}[58]$ orientation at the same fixed misorientations. Comparison of these plots shows that in general, when the energy is low, the population is high.

The preference for (100) planes and the relatively higher population of boundaries with lower energies is actually a characteristic of the entire data set, not just of the points shown in Fig. 10 [44]. To illustrate this, the relative areas $(\lambda)$ and the minimum inclination of the boundary normal from the $\langle 100\rangle$ direction $\left(\theta_{100}\right)$ were determined for every observed boundary. The energy was then discretized into equal partitions, and the mean and standard deviation of $\ln (\lambda+1)$ and $\theta_{100}$ were determined for all of the boundaries within each partition. The results in Fig. 11 show that as $\gamma$ increases, $\theta_{100}$ increases and $\lambda$ decreases; this illustrates that the trends observed in Fig. 10 persist throughout the data set.

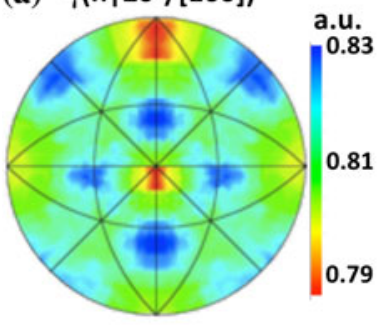

(b) $\gamma\left(\mathrm{n} \mid 20^{\circ} /[110]\right)$

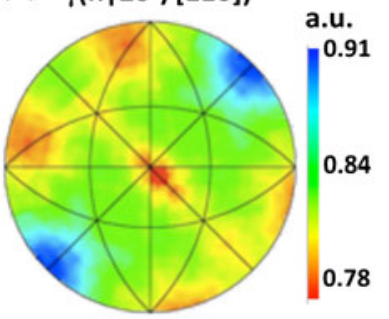

(c) $\gamma\left(\mathrm{n} \mid 20^{\circ} /[111]\right)$

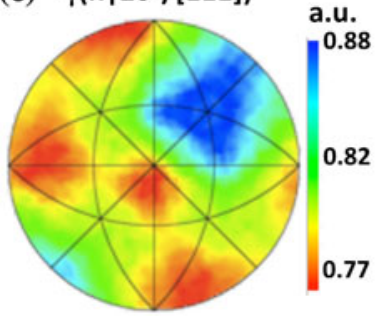

(a) $\gamma\left(\mathrm{n} \mid 20^{\circ} /[100]\right)$ (d) $\lambda\left(\mathrm{n} \mid 20^{\circ} /[100]\right)$

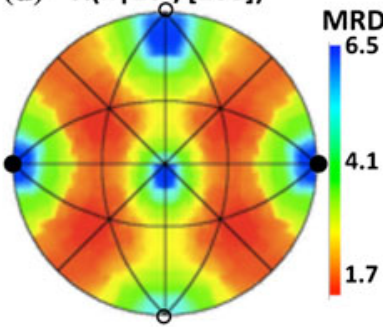

(e) $\lambda\left(\mathrm{n} \mid 20^{\circ} /[110]\right)$

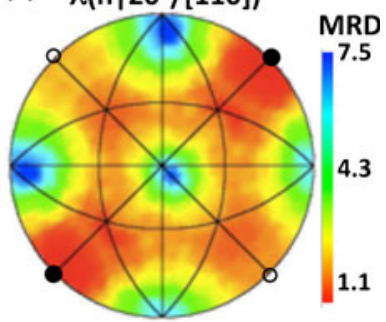

(f) $\lambda\left(n \mid 20^{\circ} /[111]\right)$

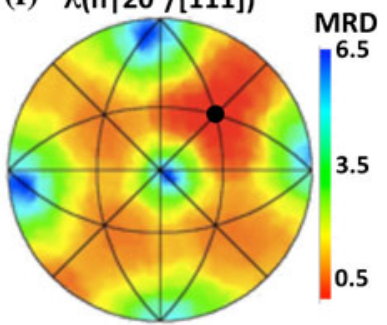

Fig. 10 Relative grain boundary energies $(\mathbf{a}-\mathbf{c})$ and grain boundary populations (d-f) for $20^{\circ}$ misorientations about the [100] (a, d), [110] $(\mathbf{b}, \mathbf{e})$, and [111] (c, f) axes for polycrystalline Ca-doped $\mathrm{MgO}$. The distributions are plotted on stereographic projections, with [001] normal to the plane of projection and [100] along the horizontal direction in the plane of projection. All of the stereograms in this paper use the same reference frame. In $\mathbf{d}-\mathbf{f}$, the orientations of the pure twist grain boundaries are marked with a filled black circle. In $\mathbf{d}$ and $\mathbf{e}$, the tilt boundaries are along the line between the unfilled circles $[43,44]$ 


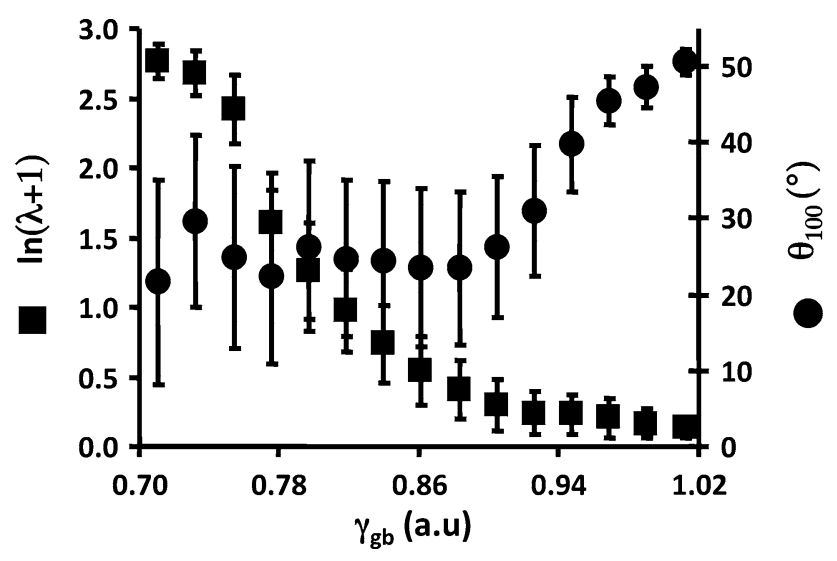

Fig. 11 The grain boundary population (squares) and the minimum inclination of the boundary normal from a $\langle 100\rangle$ direction (circles) plotted as a function of the reconstructed grain boundary energy. For each quantity, the average of all values within a range of 0.022 a.u. is represented by the point; the bars indicate one standard deviation above and below the mean [44]

Subsequent studies of other metals and ceramics suggest that similar trends persist in other systems [47, 48, 59]. In Fig. 12, the grain boundary energy as a function of grain boundary plane orientation (averaged over all lattice misorientations) is plotted in a standard stereographic triangle for $\mathrm{Y}_{2} \mathrm{O}_{3}, \mathrm{Ni}$, yttria stabilized $\mathrm{ZrO}_{2}$ (YSZ), and $\mathrm{SrTiO}_{3}$. The energy distributions are also compared to grain boundary plane distributions averaged in the same way. Note that the averaging compresses the true anisotropy. However, the basic result is that certain low index planes have lower energies and that boundaries comprised of these planes appear more frequently in the microstructure. In fact, the most commonly occurring grain boundary plane orientations are correlated with low index, low energy surface planes. This is illustrated in Fig. 13, which compares free surface energies with grain boundary populations for $\mathrm{MgO}$ (cubic) [42-44], $\mathrm{TiO}_{2}$ (rutile, tetragonal) [60], and $\mathrm{Al}_{2} \mathrm{O}_{3}$ (corundum, trigonal) [61]. On average, rather than seeking high symmetry configurations, grain boundaries tend to favor configurations in which at least one side of the interface can be terminated by a low index plane [62]. Recall that the energy cost for making a grain boundary can be thought of as the energy to create the two surfaces on either side of the interface, minus the binding energy that is recovered by bringing the two surfaces together. The observation that the total grain boundary energy is correlated to the surface energies suggests that surface energy anisotropies makes a significant contribution to the total anisotropy that, on average, is greater than the binding energy anisotropy. This is consistent with the suggestions made by Wolf and Philpot [31] based on the results of atomistic calculations.

The validity of the Read-Shockley [13] model for the energies of low misorientation angle grain boundaries has been illustrated for boundaries of a fixed misorientation angle. The current data make it possible to check the correspondence as a function of grain boundary plane. Using the Read-Shockley model and Frank's formula [15], the minimum geometrically necessary dislocation content of grain boundaries in $\mathrm{MgO}$ with a $5^{\circ}$ misorientation about [110] has been calculated and compared to the observed energy and population. This comparison, shown in Fig. 14, demonstrates qualitative agreement between the ReadShockley model and the observed grain boundary plane orientation dependence of the energy [44]. While there is not a perfect one-to-one correlation, the most important trends are reproduced in the energies, relative areas, and density of geometrically necessary dislocations: the maximum energy and dislocation density are at the pure twist location and there is a range of low energies that correspond to low dislocation densities along the axis of tilt grain boundaries.

Examination of the data for coincident site lattice misorientations confirms the findings of earlier work that concludes that while interface coincidence sometimes corresponds to low energy, it is not a good predictor of low energy boundaries. As an example, we can consider the observations for the $\Sigma 5$ pure tilt grain boundary in $\mathrm{MgO}$, shown in Fig. 15 [44]. The boundaries that couple the highest population and the lowest energy are asymmetric tilt boundaries of the type $\{100\} /\{430\}$. At the positions of the coherent, high coincidence symmetric tilts, $\{120\} /$ $\{120\}$, the population reaches a minimum and the energy reaches a maximum. This indicates that asymmetric boundaries with $\{100\}$ planes are favored over the high coincidence boundaries. Similar conclusions were reached in the analysis of data from $\mathrm{Al}$ [63] and $\mathrm{SrTiO}_{3}$ [64]. In conclusion, the current findings suggest that low energy, low index grain boundary planes are good predictors of relatively low energy boundaries, while interface coincidence is not.

Atomistic simulations have recently been used to calculate grain boundary energies in $\mathrm{Cu}, \mathrm{Al}, \mathrm{Ni}$, and $\mathrm{Au}[65$, 66]. These new calculations cover the 388 highest symmetry boundaries and, therefore, represent a more complete sampling of the entire space of grain boundary types than was available in the past. This work as led to a number of important conclusions that are consistent with the experimental observations. First, grain boundaries with the same misorientation, but different grain boundary plane orientations may have very different energies. This leads to the observation that disorientation angle is not a good predictor of grain boundary energy. Similarly, boundary coincidence is also not a good predictor of grain boundary energy. When the calculated boundary energies are plotted as a function of $\Sigma$, there is no correlation and at any fixed value of $\Sigma$, the range of energies is nearly as wide as the total anisotropy [66]. 
Fig. 12 The relative areas of grain boundary planes (grain boundary plane distribution, GBPD) and relative grain boundary energies (GBED) for four materials, as a function of grain boundary plane orientation, without consideration of misorientation. In all four materials, there is an approximate inverse relation between the GBPD and the GBED. a $\mathrm{Y}_{2} \mathrm{O}_{3}$ [48], b $\mathrm{Ni}$ [47], c YSZ, and d $\mathrm{SrTiO}_{3}$ (a)
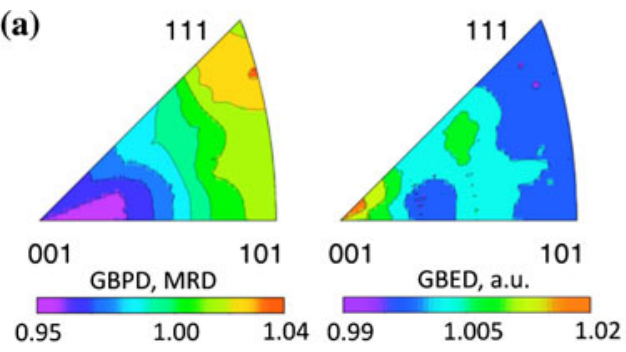

(b)
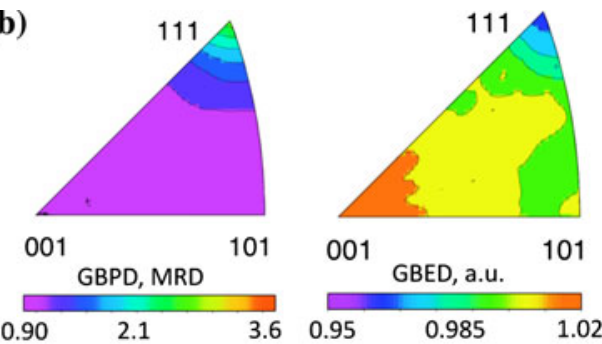

(c)
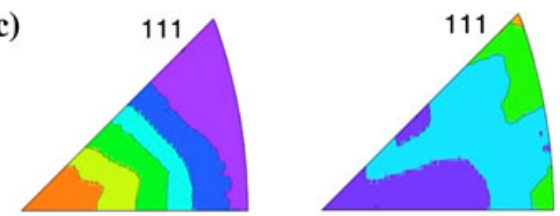

(d)
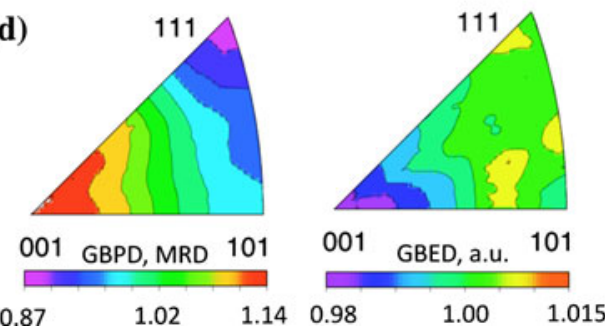

The calculations also reveal that when all of the grain boundary energies of crystallographically identical grain boundaries in different metals are compared, there is a strong linear correlation. For a pair of metals, the ratio of the energies of the vast majority of boundaries is very nearly equal to the ratio of $a_{0} G$, where $a_{0}$ is the lattice constant and $G$ is the shear modulus [65]. Recall that the rough estimate for the grain boundary energy discussed in "Introduction" section also scaled with the elastic properties of the material. For boundaries with stacking fault character, the boundary energy ratio is closer to the stacking fault energy ratio. One possible implication of this observation is that there is a single, scalable, grain boundary energy distribution for any given structure type. Based on the fact that the energies scale with the shear modulus, the second implication is that a dislocation model for the grain boundary might be valuable in predicting the energy.

The large catalog of grain boundary energies provided by Olmsted and co-workers [65, 66] allows a detailed comparison of experimentally observed and calculated grain boundary energies. $\mathrm{Ni}$ is one of the materials for which the relative grain boundary energies have been measured over all five parameters and this is also one of the materials for which grain boundary energies have been calculated [47]. A recent comparison of these data revealed the benefits and limitations of both methods [46]. The basic result was that for grain boundaries that are frequently observed in the experiment, the experimental energies and calculated energies are strongly correlated. This is illustrated in Fig. 16, where the calculated energies for $\Sigma 3$ grain boundaries in $\mathrm{Ni}$ are compared to the observed energies for the same grain boundaries. With the exception of the two outliers (circled in red), there is a very strong correlation between the results. The unweighted correlation coefficient is 0.71 and the population weighted correlation coefficient is 0.95 ; if the two outliers are eliminated, the unweighted correlation coefficient is 0.80 and the population weighted correlation coefficient is 0.98 . It has previously been mentioned that there is a strong inverse correlation between energy and populations. Because the maxima (outliers) in the experimental energy distribution (Fig. 16b) do not correspond to minima in the experimental population distribution, these points are considered questionable.

Even with some outliers, the correspondence between calculated and observed grain boundary energies is gratifying. One of the disadvantages of the experiment is that if a grain boundary does not appear frequently in the polycrystal and is not sufficiently sampled in the data set, the energy cannot be reliably determined. The simulations are obviously not subject to this constraint. On the other hand, if a boundary appears frequently enough in the microstructure, its energy can be determined by the experiment, regardless of its symmetry or the size of its repeat unit. However, the calculation has an upper limit in the size of the simulation cell and is not able to determine the energies of low symmetry boundaries with large repeat units.

\section{Grain boundary complexions}

Grain boundary composition is known to affect grain boundary energy and it has been shown that the grain boundary population is influenced by segregating impurities [60, 67]. Nevertheless, it had generally been assumed that the properties of grain boundaries changed continuously with temperature and composition. Over the last several years, a body of evidence has been published demonstrating that grain boundaries can undergo discontinuous changes in structure and composition and that these 
Fig. 13 Measured surface energies for a $\mathrm{MgO}$ [50], b $\mathrm{TiO}_{2}$ [60], and $\mathbf{c ~} \mathrm{Al}_{2} \mathrm{O}_{3}$ [61] compared to grain boundary plane orientation distributions, independent of misorientation, for $\mathbf{d ~} \mathrm{MgO}$ [43], e $\mathrm{TiO}_{2}$ [60], and $\mathbf{f} \mathrm{Al}_{2} \mathrm{O}_{3}$. In each case, the lowest surface energy orientations correspond to larger than expected populations
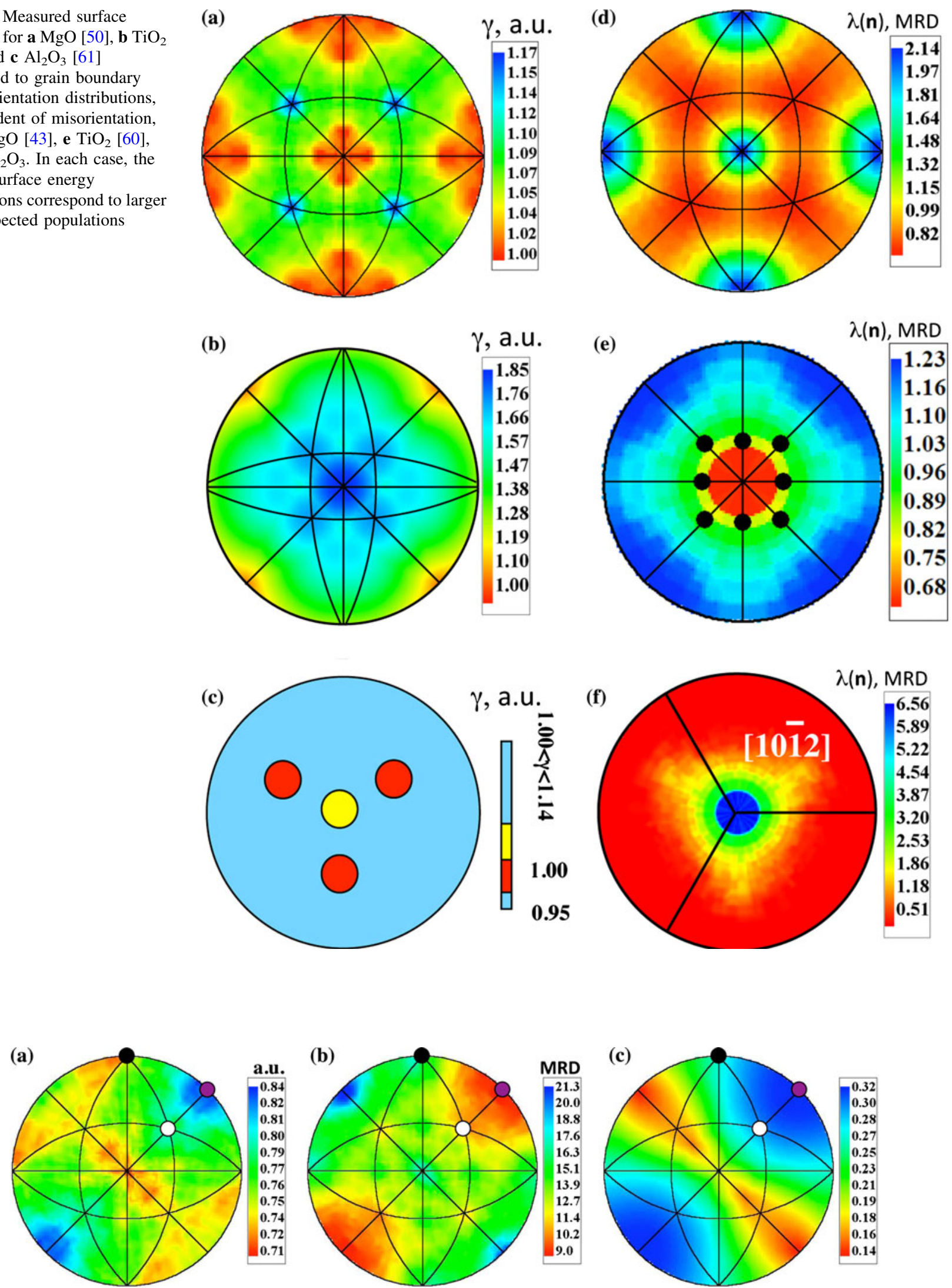

Fig. 14 a Grain boundary energies, b the observed population, and $\mathbf{c}$ geometrically necessary dislocation densities for a $5^{\circ}$ misorientation about [110] in $\mathrm{MgO}$ [44] 


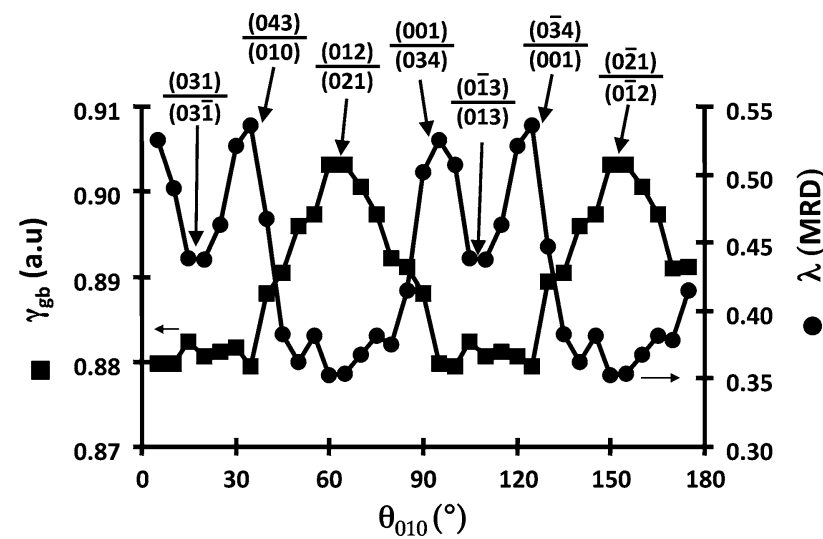

Fig. 15 Comparison of the reconstructed energies (squares) and the observed population (circles) for $\Sigma 5$ tilt boundaries. The quantities are plotted in $5^{\circ}$ intervals as a function of the angle between the boundary plane normal and $(010) /(04 \overline{3})$. For reference, the location of the symmetric tilts, both the $\{310\}$ and $\{210\}$ types, as well as the asymmetric boundaries terminated by $\{100\}$ planes, are indicated. Note that the function has mirror symmetries at the symmetric tilt orientations, $\{210\}$ and $\{310\}$. The deviations from this are due to the relatively large intervals of the plotted points [44]

transitions can be associated with transitions in mobility and energy. These grain boundary states have been referred to by some authors as "complexions" [68-73]. The breakthrough experimental studies by Dillon and Harmer $[69,74,75]$ focused on alumina with controlled impurity concentrations. High resolution microscopy was used to demonstrate that boundaries could be pure, could have a single adsorbed monolayer of solute, or an adsorbed bilayer of solute. Other boundaries had multilayer adsorption, thin intergranular wetting films of constant thickness, or films of arbitrary thickness. While some of these boundary structures had been observed previously, the breakthrough was associating them with very different grain boundary mobilities and observing that they could co-exist in the same microstructure. Based on these findings, Harmer and Dillon $[76,77]$ have described a plausible mechanism for abnormal grain growth.

More recent work has demonstrated that grain boundaries with different complexions can have different energies [78, 79]. For example, the data in Fig. 17 shows the relative grain boundary energy distributions for two subsets of grain boundaries in the same $100 \mathrm{ppm} \mathrm{Nd-doped} \mathrm{alu-}$ mina [79]. These measurements were derived from thermal grooves according to Eq. 5. In this case, the crystallographic characteristics of the junctions were not considered and we are concerned only with the distribution derived by averaging over more than 200 boundaries. The first subset of boundaries surrounds small grains growing normally (NGG) and the second subset surrounds grains growing abnormally (AGG). The grain boundaries growing normally are known to have a monolayer of adsorbed $\mathrm{Nd}$ and

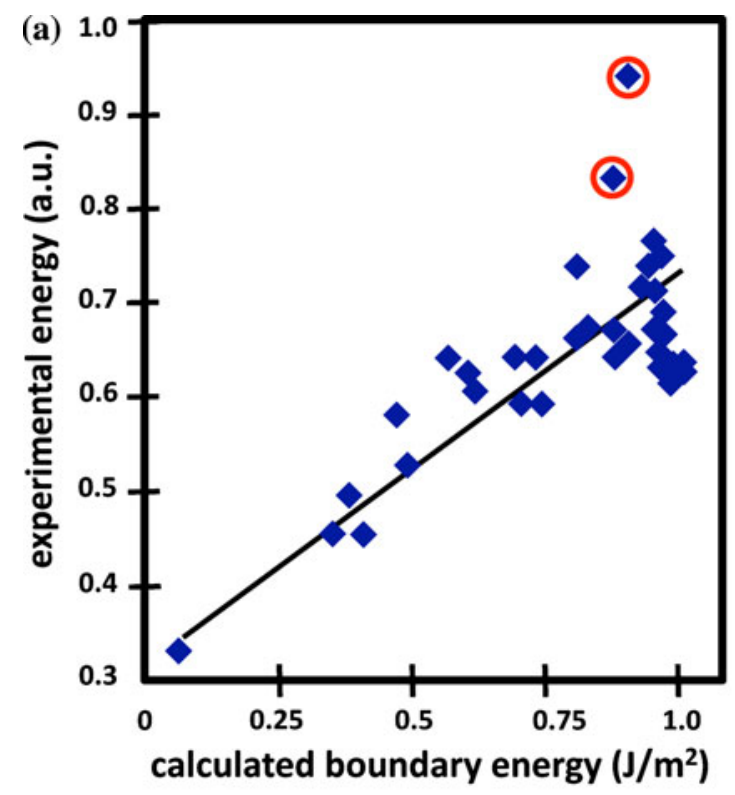

(b)
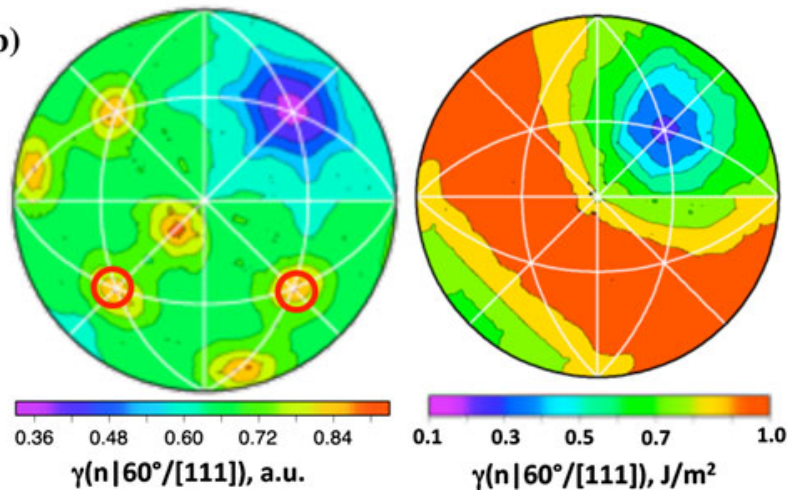

Fig. 16 a The relationship between the experimental and calculated grain boundary energy for $\Sigma 3$ grain boundaries in Ni. The two circled points are outliers [46]. b The experimental energy distribution, the circled positions correspond to the circled points in a [47]. $\mathbf{c}$ Contour plot of calculated energies for comparison to $\mathbf{b}$

the grains growing abnormally have a bilayer of adsorbed $\mathrm{Nd}$ and a greater mobility. The results clearly show that the boundaries enriched with $\mathrm{Nd}$ have a lower average energy than those with less Nd. One question is why the complexion transition nucleates at some grain boundaries, but not at all boundaries. It is possible that it is the highest energy boundaries that preferentially transform, as discussed by Wynblatt and Chatain [73], so an understanding of grain boundary energy anisotropy is necessary to develop a mechanism for complexion transitions. In this same work, it was noted that complexion transitions need not be of first order and some complexions can form without an activation barrier [73].

One outstanding question has been, why do some boundaries enriched in solute and undergo complexion transitions while others simply deposit the solute in the 


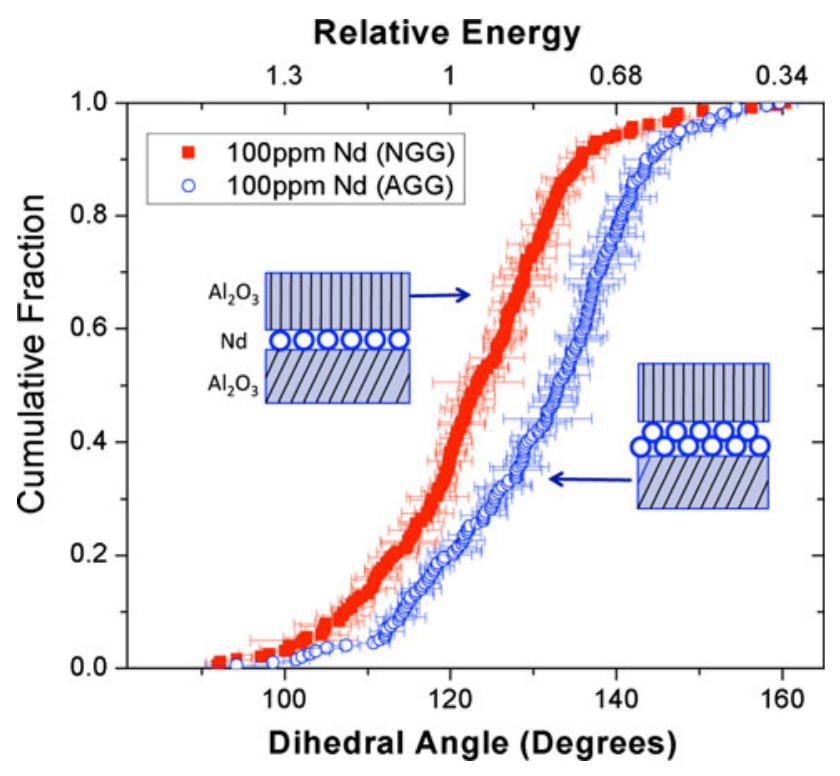

Fig. 17 Cumulative distribution of dihedral angles in neodymiadoped alumina annealed at $1400{ }^{\circ} \mathrm{C}$ with normal (complexion I) and abnormal (complexion III) grain boundaries. Insets schematically illustrate the boundary structure of the two complexions [79]

form of precipitate phases? A recent experiment has explored this issue and found that the relative energy of the interface between a precipitate and the host lattice is an indicator of whether or not a complexion transition will occur [80]. Chemistries that produce low-energy interphase boundaries tend to suppress complexion transitions, while those nucleating precipitates with high interfacial energies promote them. This may be explained in the context of a phase selection competition in which the activation barrier to the complexion transition and precipitation compete with one another. The interphase boundary energies tend to be intermediate to the energies of the grain boundaries in the component systems. These facts lead to a proposed selection criterion for additives based on knowledge of the interfacial energies. Namely, complexion transitions should be sought in systems where the solute strongly segregates to the boundary and where precipitates with coherent, low energy interfaces do not form.

\section{Future prospects for grain boundary energy studies}

There has been significant progress in understanding the anisotropy of grain boundary energies since the initial experimental studies by Dunn and Leonetti [33]. One significant opportunity for further study is to explore the relationship between grain boundary energy and population. The measurements of the grain boundary population are more accurate than measurements of the energy. If there is a fixed relationship between the two, it would be possible to calculate the energy directly from the population measurement, rather than fitting the Herring equation to the observed geometries of the triple junctions. This would allow the generation of a greater quantity of higher quality grain boundary energy data. This has been suggested by Gruber et al. [81, 82] and demonstrated in onedimension, but the method has not yet been extended to all five crystallographic dimensions.

A related question is whether or not grain boundary plane distributions represent grain boundary Wulff shapes. In other words, if the energy and population are inversely correlated, it is possible that the statistical grain boundary distribution is a measure of the grain boundary energy anisotropy just as the shape a small crystal in equilibrium is a measure of the surface energy anisotropy. Although grain boundaries are moving at the high temperatures where the grain boundary distribution is determined, local equilibrium is obtained at the triple junctions and this may be the feature that creates statistically equivalent grain boundary population distributions at different grain sizes [83]. If so, this would also represent a path toward measuring grain boundary energies.

As mentioned previously, there is evidence that the grain boundary energy anisotropies of isostructural materials are roughly the same. If we can understand this scaling and the bounds on the correlation, it will be of great value to generalize the past findings. This will require the exploration of materials beyond fcc metals. Such a finding could be applied to grain boundary engineering [84], where changes in the grain boundary character distribution with sequential thermomechanical processing are thought to be closely related to the grain boundary energy distribution [85-87].

Finally, chemical effects on the grain boundary energy anisotropy remain largely unexplored. This is particularly important with respect to understanding complexion transitions. Because of the effect that complexion transitions have on grain boundary mobility, they offer the possibility of controlling and designing new multicomponent materials. However, our currently incomplete knowledge of the mechanisms governing complexion transitions makes it impossible to predict their occurrence. Grain boundary energy measurements are likely to play an important role in this emerging research area.

\section{Summary}

It has been nearly a century since Rosenhain and Ewan [11] first speculated about the nature of intergranular cohesion. While their "amorphous cement" model has sometimes been looked upon as being a naïve product of the times, it is not entirely inaccurate. Recent studies of doped aluminas 
have definitively shown that disordered regions can exist in the intergranular region [72]. While Rosenhain and Ewan's [11] model was basically speculation, we now have the benefit of almost 100 years of observations to consider what we know about grain boundaries. Based on the accumulated data, there are several fundamental characteristics of the grain boundary energy anisotropy that appear to be definitively established, and these are enumerated below:

1. The energy anisotropy that results from variations in the grain boundary plane orientation are greater than the anisotropy that results from variations in the lattice misorientation.

2. The Read-Shockley model for the energies of small misorientation angle grain boundaries provides reliable predictions for relative grain boundary energies.

3. Models based lattice or boundary coincidence are not good predictors of grain boundary energies.

4. There is an inverse correlation between the grain boundary energy and the grain boundary population.

5. Grain boundaries comprised of low energy surfaces have relatively low energies.

6. Isostructural materials have similar grain boundary energy anisotropies.

Acknowledgements The work was supported by the MRSEC program of the National Science Foundation under Award Number DMR-0520425. The author acknowledges collaboration with S.J. Dillon, M.P. Harmer, J. Gruber, C.S. Kim, S.B. Lee, J. Li, H.M. Miller, T. Sano, D.M. Saylor, A.D. Rollett, and P. Wynblatt in studies of grain boundary energies at Carnegie Mellon University.

\section{References}

1. Brandon D (2010) Mater Sci Technol 26:762. doi:10.1179/0267 08310x12635619987989

2. Harmer MP (2010) J Am Ceram Soc 93:301. doi:10.1111/J.15512916.2009.03545.X

3. Rohrer GS (2005) Ann Rev Mater Res 35:99. doi:10.1146/ annurev.matsci.33.041002.094657

4. Rohrer GS, Saylor DM, El Dasher B, Adams BL, Rollett AD, Wynblatt P (2004) Z Metallk 95:197

5. Rohrer GS (2011) J Am Ceram Soc 94:633. doi:10.1111/j.15512916.2011.04384.x

6. Sutton AP, Balluffi RW (1987) Acta Metall 35:2177

7. Mullins WW (1963) Metal surfaces. American Society for Metals, Metals Park, OH

8. Mullins WW (1956) J Appl Phys 27:900

9. Smith CS (1948) Trans AIMME 175:15

10. Dillon SJ, Rohrer GS (2009) Acta Mater 57:1. doi:10.1016/j. actamat.2008.08.062

11. Rosenhain W, Ewen D (1912) J Inst Met 8:149

12. Hargreaves F, Hills RJ (1929) J Inst Met 41:257

13. Read WT, Shockley W (1950) Phys Rev 78:275

14. Gjostein NA, Rhines FN (1959) Acta Metall 7:319

15. Frank FC (1950) In: Koehler JS (ed) A symposium on the plastic deformation of crystalline solids. Office of Naval Research, Pittsburgh, PA
16. Read WT, Shockley W (1952) Imperfections in nearly perfect crystals. Wiley, New York

17. Kronberg ML, Wilson FH (1949) Trans AIMME 185:501

18. Duparc O (2011) J Mater Sci 46:4116. doi:10.1007/s10853$011-5367-1$

19. Friedel G (1920) Bulletin de la Société Française de Minéralogie 43:246

20. Aust KT, Rutter JW (1959) Trans AIMME 215:119

21. Brandon DG (1966) Acta Metall 14:1479

22. Brandon DG, Ralph B, Ranganathan S, Wald MS (1964) Acta Metall 12:813

23. Goodhew PJ, Tan TY, Balluffi RW (1978) Acta Metall 26:557

24. Chaudhari P, Matthews JW (1971) J Appl Phys 42:3063

25. Ashby MF, Spaepen F, Williams S (1978) Acta Metall 26:1647

26. Gleiter H (1982) Mater Sci Eng 52:91

27. Weins M, Chalmers B, Gleiter H, Ashby M (1969) Scr Metall 3:601

28. Wolf D (1990) J Mater Res 5:1708

29. Wolf D (1990) Acta Metall Mater 38:781

30. Wolf D (1990) Acta Metall Mater 38:791

31. Wolf D, Phillpot S (1989) Mater Sci Eng A 107:3

32. Herring C (1951) In: Kingston WE (ed) The physics of powder metallurgy. McGraw-Hill, New York

33. Dunn CG, Lionetti F (1949) Trans AIMME 185:125

34. Hasson G, Herbeuva I, Boos JY, Biscondi M, Goux C (1972) Surf Sci $31: 115$

35. Hasson GC, Goux C (1971) Scr Metall 5:889

36. Saylor DM, Morawiec A, Adams BL, Rohrer GS (2000) Interface Sci 8:131

37. Mullins WW (1957) J Appl Phys 28:333

38. Saylor DM, Rohrer GS (1999) J Am Ceram Soc 82:1529

39. Schwartz AJ, Kumar M, Adams BL, Field DP (2009) Electron backscatter diffraction in materials science. Springer, New York

40. Rollett AD, Lee SB, Campman R, Rohrer GS (2007) Ann Rev Mater Res 37:627. doi:10.1146/annurev.matsci.37.052506. 084401

41. Adams BL, Wright SI, Kunze K (1993) Met Trans A 24:819

42. Saylor DM, Morawiec A, Rohrer GS (2002) J Am Ceram Soc 85:3081

43. Saylor DM, Morawiec A, Rohrer GS (2003) Acta Mater 51:3663. doi:10.1016/S1359-6454(03)00181-2

44. Saylor DM, Morawiec A, Rohrer GS (2003) Acta Mater 51:3675. doi:10.1016/S1359-6454(03)00182-4

45. Morawiec A (2000) Acta Mater 48:3525

46. Rohrer GS, Holm EA, Rollett AD, Foiles SM, Li J, Olmsted DL (2010) Acta Mater 58:5063. doi:10.1016/j.actamat.2010.05.042

47. Li J, Dillon SJ, Rohrer GS (2009) Acta Mater 57:4304. doi: 10.1016/j.actamat.2009.06.004

48. Dillon SJ, Rohrer GS (2009) J Am Ceram Soc 92:1580. doi: 10.1111/j.1551-2916.2009.03064.x

49. Sano T, Saylor DM, Rohrer GS (2003) J Am Ceram Soc 86:1933

50. Saylor DM, Rohrer GS (2001) Interface Sci 9:35

51. Miura H, Kato M, Mori T (1994) J Mater Sci Lett 13:46

52. Skidmore T, Buchheit RG, Juhas MC (2004) Scr Mater 50:873. doi:10.1016/j.scriptamat.2003.12.004

53. McLean M (1973) J Mater Sci 8:571. doi:10.1007/BF00550462

54. Tschopp MA, McDowell DL (2007) Philos Mag 87:3871. doi: $10.1080 / 14786430701455321$

55. Dhalenne G, Revcolevschi A, Gervais A (1979) Physica Status Solidi A 56:267

56. Readey DW, Jech RE (1968) J Am Ceram Soc 51:201

57. Dhalenne G, Dechamps M, Revcolevschi A (1982) J Am Ceram Soc 65:C11

58. Kimura S, Yasuda E, Sakaki M (1986) Yogyo-Kyokai-Shi 94:795

59. Rohrer GS, Li J, Lee S, Rollett AD, Groeber M, Uchic MD (2010) Mater Sci Technol 26:661. doi:10.1179/026708309X1 2468927349370 
60. Pang Y, Wynblatt P (2006) J Am Ceram Soc 89:666. doi: 10.1111/j.1551-2916.2005.007993x

61. Kitayama M, Glaeser AM (2002) J Am Ceram Soc 85:611

62. Saylor DM, El Dasher B, Pang Y et al (2004) J Am Ceram Soc 87:724

63. Saylor DM, El Dasher BS, Rollett AD, Rohrer GS (2004) Acta Mater 52:3649. doi:10.1016/j.actamat.2004.04.018

64. Saylor DM, El Dasher B, Sano T, Rohrer GS (2004) J Am Ceram Soc 87:670

65. Holm EA, Olmsted DL, Foiles SM (2010) Scr Mater 63:905. doi: 10.1016/j.scriptamat.2010.06.040

66. Olmsted DL, Foiles SM, Holm EA (2009) Acta Mater 57:3694. doi:10.1016/j.actamat.2009.04.007

67. Papillon F, Rohrer GS, Wynblatt P (2009) J Am Ceram Soc 92:3044. doi:10.1111/j.1551-2916.2009.03327.x

68. Baram M, Chatain D, Kaplan WD (2011) Science 332:206. doi: 10.1126/science. 1201596

69. Dillon SJ, Tang M, Carter WC, Harmer MP (2007) Acta Mater 55:6208. doi:10.1016/j.actamat.2007.07.029

70. Tang M, Carter WC, Cannon RM (2006) Phys Rev Lett 97:4. doi: 07550210.1103/PhysRevLett.97.075502

71. Tang M, Carter WC, Cannon RM (2006) J Mater Sci 41:7691. doi:10.1007/s10853-006-0608-4

72. Tang M, Carter WC, Cannon RM (2006) Phys Rev B 73:14. doi: 02410210.1103/PhysRevB.73.024102

73. Wynblatt P, Chatain D (2008) Mater Sci Eng A 495:119. doi: 10.1016/j.msea.2007.09.091

74. Dillon SJ, Harmer MP (2007) Acta Mater 55:5247. doi: 10.1016/j.actamat.2007.04.051
75. Dillon SJ, Harmer MP (2007) J Am Ceram Soc 90:996. doi: 10.1111/j.1551-2916.2007.01512.x

76. Dillon SJ, Harmer MP (2008) J Am Ceram Soc 91:2304. doi: 10.1111/j.1551-2916.2008.02454.x

77. Dillon SJ, Harmer MP (2008) J Am Ceram Soc 91:2314. doi: 10.1111/j.1551-2916.2008.02432.x

78. Dillon SJ, Miller H, Harmer MP, Rohrer GS (2010) Int J Mater Res 101:50. doi:10.3139/146.110253

79. Dillon SJ, Harmer MP, Rohrer GS (2010) J Am Ceram Soc 93:1796. doi:10.1111/j.1551-2916.2010.03642.x

80. Dillon SJ, Harmer MP, Rohrer GS (2010) Acta Mater 58:5097. doi:10.1016/j.actamat.2010.05.045

81. Gruber J, Rollett AD, Rohrer GS (2010) Acta Mater 58:14. doi: 10.1016/j.actamat.2009.08.032

82. Gruber J, Miller HM, Hoffmann TD, Rohrer GS, Rollett AD (2009) Acta Mater 57:6102. doi:10.1016/j.actamat.2009.08.036

83. Gruber J, George DC, Kuprat AP, Rohrer GS, Rollett AD (2005) Scr Mater 53:351. doi:10.1016/j.scriptamat.2005.04.004

84. Watanabe T (2011) J Mater Sci 46:4095. doi:10.1007/s10853011-5393-z

85. Randle V, Rohrer GS, Miller HM, Coleman M, Owen GT (2008) Acta Mater 56:2363. doi:10.1016/j.actamat.2008.01.039

86. Rohrer GS, Randle V, Kim CS, Hu Y (2006) Acta Mater 54:4489. doi:10.1016/j.actamat.2006.05.035

87. Kim CS, Hu Y, Rohrer GS, Randle V (2005) Scr Mater 52:633. doi:10.1016/j.scriptamat.2004.11.025 